\section{Array Reactors for Parallel Synthesis}

\author{
Philip F. Hughes,* Thomas H. Graham, ${ }^{\dagger}$ and
} Jose S. Mendoza

Lilly RTP Labs, Lilly Research Laboratories, Eli Lilly \& Company, 20 T. W. Alexander Drive, Research Triangle Park, North Carolina 27709

\section{Received January 30, 2004}

The development of high-throughput screening (HTS) in the past decade generated the capacity to quickly evaluate large numbers of compounds in a variety of assay methodologies. ${ }^{1}$ In many organizations, this capacity easily exceeded the size of internal compound collections. Efficient utilization of this HTS resource drove the need for an analogous increase in the rate of compound synthesis. This, in turn, drove the development of technologies and processes, with the goal of multiplying the capacity of classical one-at-atime organic synthesis. ${ }^{2}$

One approach, which utilizes both combinatorial and parallel efficiencies, is to run reactions in an array. Over the past few years, we have worked to develop a solution-phase array reactor, which mimics the simplicity but can accommodate the functionality of the classic round-bottom flask. In other words, it should be simple but, with the addition of optional pieces, gain functionality. Although there are a number of commercial apparatuses ${ }^{3}$ approaching this ideal, we felt that a better (lighter, simpler, and less expensive) solution could be devised. We describe here the current incarnation of our development efforts.

While developing an array reactor, we adhered to a few useful guiding design constraints to enforce simplicity and flexibility. The device should be lightweight, should use commodity, disposable glass for reaction vessels, should fit a microtiter ${ }^{4}$ footprint, and should enable a wide range of reaction conditions. The general design principles should allow construction of devices with numerous small reaction vessels or fewer large reaction vessels. Finally, the device should integrate well with standard laboratory equipment, such as hotplate/stirrers, in addition to equipment more associated with parallel processes, such as filter plates or liquid handling robots.

We have developed three array reactors that adhere to these design constraints; the SynthArray-96, the SynthArray24, and the SynthArray-6, which have 96 2-mL, 24 8-mL, and $640-\mathrm{mL}$ reaction vessels, respectively. The following description will focus on the SynthArray-24, since this reactor has been most thoroughly developed, tested, and used.

* Corresponding author address: InnovaSyn LLC, 627 Arlington Street, Chapel Hill, NC, 27514. E-mail: hat@nc.rr.com.

$\dagger$ Current address: Department of Chemistry, University of Pittsburgh, 234 Chevron Science Center, Pittsburgh, PA 15260.

$\doteqdot$ Current address: Amphora Discovery Corporation, PO Box 12169, Research Triangle Park, NC 27709-2169.
The SynthArray-24 consists of six pieces. Shown ${ }^{5}$ in Figure 1 are (1) the tube holder, (2) the sleeve, (3) the solid top, (4) the top with holes, (5) the heater block/alignment guide, and (6) the heat transfer block. The tube holder 1 is machined from Teflon, and the other parts are made from aluminum. Drawings with measurements are included as Supporting Information.

The central piece of the SynthArray-24 is the Teflon tube holder 1 . The holder has a centered $4 \times 6$ array of 24 holes (18-mm spacing) with embedded O-rings that hold reaction tubes $^{6}$ and provide a gastight seal. There are also four holes at the corners through which screws can pass. The reaction vessels are $13 \times 100 \mathrm{~mm}$ screw-cap culture tubes. The tubes can be inserted into the holder by hand or, more conveniently, by pressing the holder onto the tubes with an arbor press. This (Figure 2a) is the simplest configuration and is adequate for many reactions. Addition of 24 stir bars provides ample stirring when the reactor is placed on a conventional laboratory magnetic stirrer. Caps can be placed on the tubes during a reaction and for storage.

For a controlled atmosphere, an aluminum sleeve, 2, with a Leur gas inlet in the side, is snapped onto the top side of the Teflon holder, and a solid plate top, $\mathbf{3}$, held in place with four screws and nuts is added (Figure $2 b$ ). If needle access to the tubes is needed to allow addition of reagents under inert atmosphere, a Teflon-faced silicone sheet and a plate with 24 holes, $\mathbf{4}$, aligned with the tubes is placed on top instead (Figure 3a).

One advantage of holding the tubes at the top is that, analogous to a round-bottom flask, one is not limited in the way temperature is controlled at the bottom of the reactor. Thus, the reactor can be easily placed in a variety of heating or cooling baths or blocks. Another advantage is that, because the reactor is not coupled to the heater, the reactor can be quickly removed from the heat source. Our preferred method of heating is to place the reactor in a heater block/alignment guide, $\mathbf{5}$, on a thermostatically controlled hotplate (Figure $3 \mathrm{~b})$. The heater block is a half-inch aluminum block with 24 holes drilled through for the reactor tubes as well as a hole for insertion of a thermocouple probe.

The aluminum heater block/alignment guide can serve two other purposes. The block has four tapped screw holes on the corners so that it can slide up the tubes and act as a rigid receptor for the screws holding down the top. This forms a tighter seal, which is useful for reactions requiring better atmospheric integrity. Atmospheric hydrogenations (balloon pressure) have been accomplished using this configuration (Figure 4a), which allows for alternate vacuum and gas purging. In addition, because the block has the outside dimensions of a microtiter plate, it is used to align the reactor block precisely when placed on a liquid handling robot.

Another optional piece of the reactor is the heat transfer block, $\mathbf{6}$. This is an aluminum block similar to the heater block except that a channel runs around the perimeter of the block with access at the four corners. By sealing one pair of 

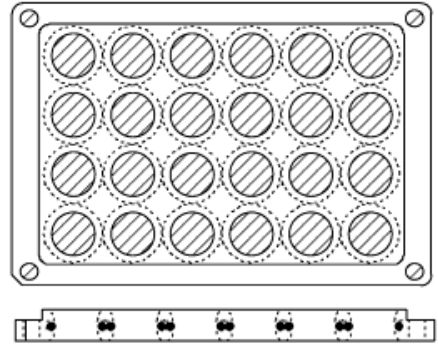

1

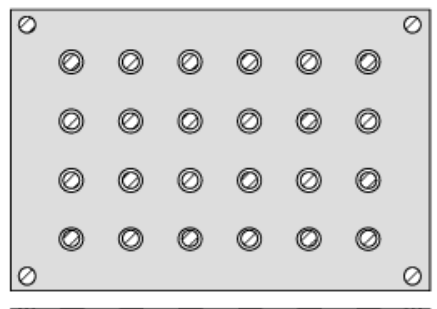

4

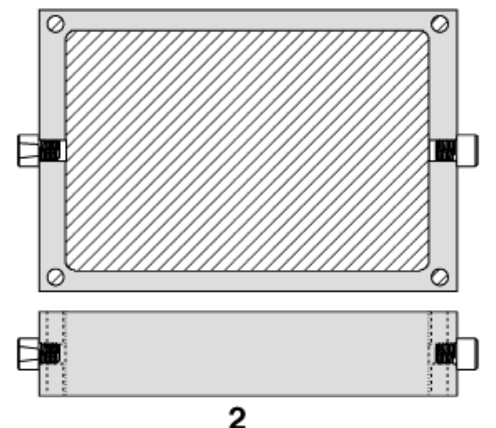

2

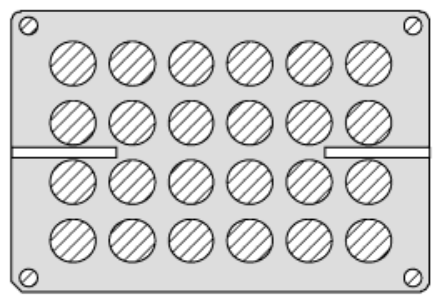

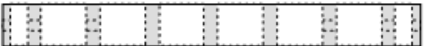

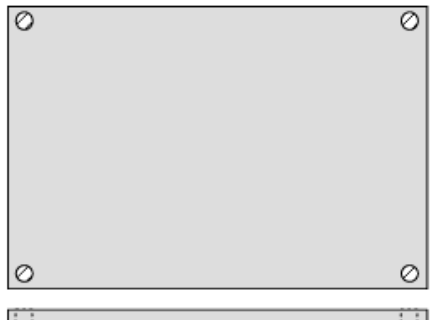

3

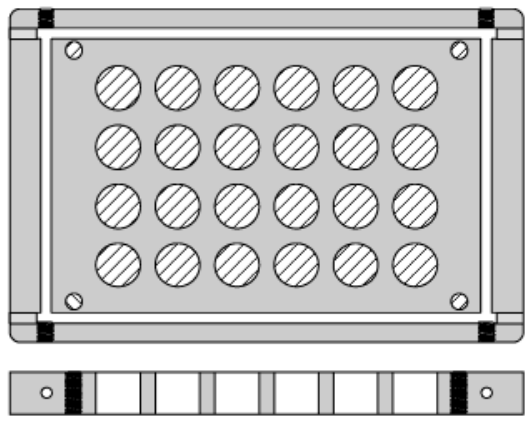

6

Figure 1. SynthArray-24 reactor parts (1) tube holder, (2) sleeve, (3) solid top, (4) top with holes, (5) heater block/alignment guide, and (6) heat transfer block.

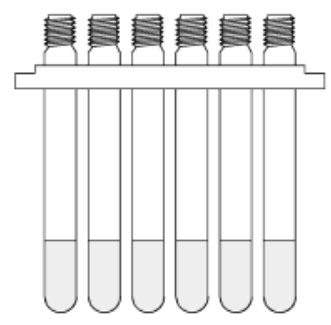

a. Tube Holder.

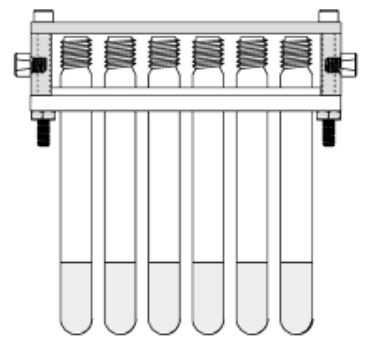

b. Inert Atmosphere.

Figure 2. (a) Tube holder, (b) inert atmosphere.

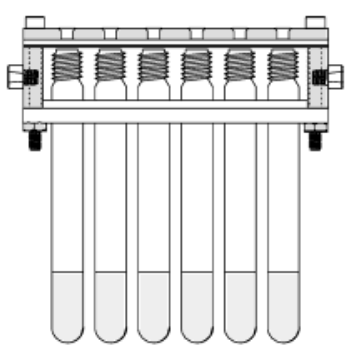

a. With Septa Top

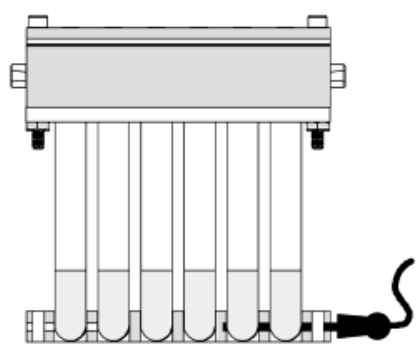

b. With Heater Block

Figure 3. (a) With septa top, (b) with heater block.

diagonal holes and plumbing the opposite holes, one can use the block as a condenser when screwed to the base of the tube holder (Figure 4b). We have been able to vigorously reflux solvents (e.g., $\mathrm{CH}_{2} \mathrm{Cl}_{2}, \mathrm{EtOH}$, toluene) overnight without any noticeable loss of solvent. Alternatively, it can be used as a reaction heater or cooler when dropped to the bottom of the vials.

A reaction setup for reflux under an inert atmosphere (using all six parts) is shown in Figure 5a. One convenient feature of the heater block is that if short screws are put into the block, the protruding threads very nicely align the reaction block on an IKAMAG heater/stirrer. ${ }^{7}$ We have also

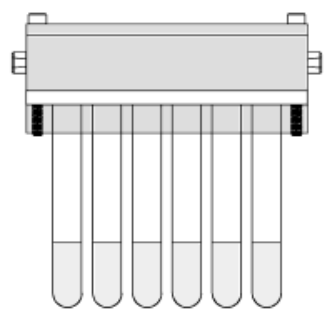

a. Hydrogenation Setup

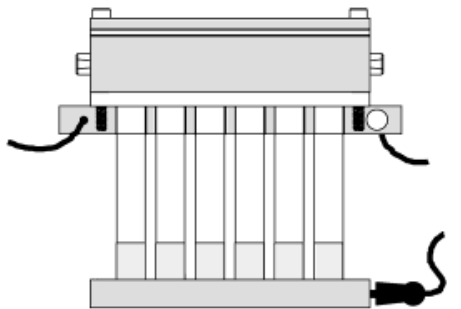

b. Reflux Setup
Figure 4. (a) Hydrogenation setup, (b) reflux setup.

developed a 24-hole funnel and a variety of solid addition plates that can deliver $150 \mu \mathrm{L}, 300 \mu \mathrm{L}, 600 \mu \mathrm{L}, 1 \mathrm{~mL}$, and $2 \mathrm{~mL}$ volumes of solid per tube (Figure $5 \mathrm{~b}$ ).

The SynthArray-6 is simply the same design as the SynthArray-24 with 6 holes designed to fit 40 - or $60-\mathrm{mL}$ EPA vials, with 36-mm spacing between wells. The holder, the heat transfer block, and the heater block are also made from thicker stock. The SynthArray-96 is designed similarly, except that the tube holder is machined from polypropylene with a lip around the top of each of the 96 holes to hold and seal around the tubes. Spacing between the tubes does not allow room for standard O-rings. The SynthArray-96 can use standard WISP autosampler vials $(8.1 \mathrm{~mm} \times 43 \mathrm{~mm})$; however, we use taller custom vials $(8.1 \mathrm{~mm} \times 80 \mathrm{~mm})$ for additional volume and efficient refluxing. Mixing in the 96well reactor can be accomplished with stir bars or by placing the reactor on a commercial orbital shaker equipped with a heater block. ${ }^{8}$ Although the designs of the 6- and 96- well reactors continue to evolve, ${ }^{9}$ efficient heating, cooling, and refluxing also work well with these systems. Photographs of both are shown in Figure 6. With all three reactors, one can replace a tube simply by pushing it out and pushing in another. All the tubes can be removed quickly by suspending the holder and pushing the tubes out with an arbor press. 


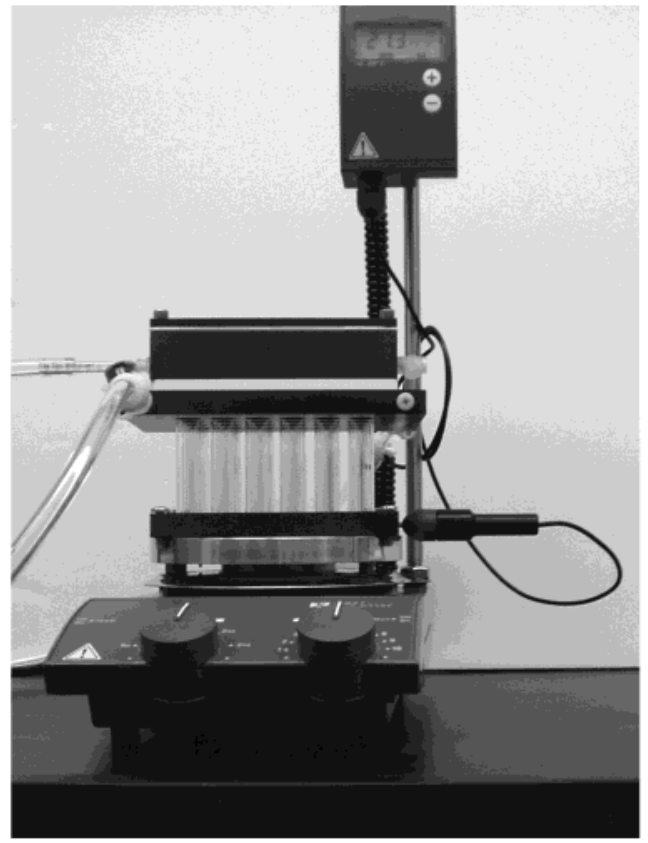

a. Complete Setup

Figure 5. (a) Complete setup, (b) solid addition plate with funnel.

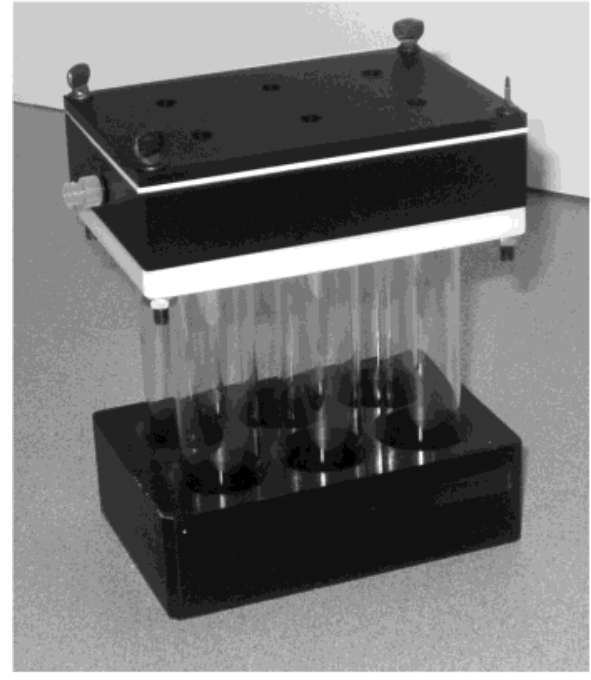

\section{SynthArray-6}

Figure 6. SynthArray-6 SynthArray-96.

We have described three simple but highly functional array reactors for parallel solution phase chemistry. Use of the reactors mimics that of a standard round-bottom flask in that function can be easily added, but only as needed, to allow a wide range of reaction conditions. We have used these reactors to run parallel reactions from -78 to $200{ }^{\circ} \mathrm{C}$ under open and inert atmosphere. The reactors have been used for numerous reactions, including reductive alkylations, Mitsunobu reactions; Suzuki, Buchwald and Heck couplings; lithium halogen exchange reactions; Grignard reactions; hydrogenations; and the Fisher indole synthesis. The replaceable glass reaction vessels are inexpensive ( $\sim$ 0.12 ea), disposable, commodity, glass vials. ${ }^{10}$ The permanent components are easily cleaned and have all gone through our glass washing service numerous times without noticeable

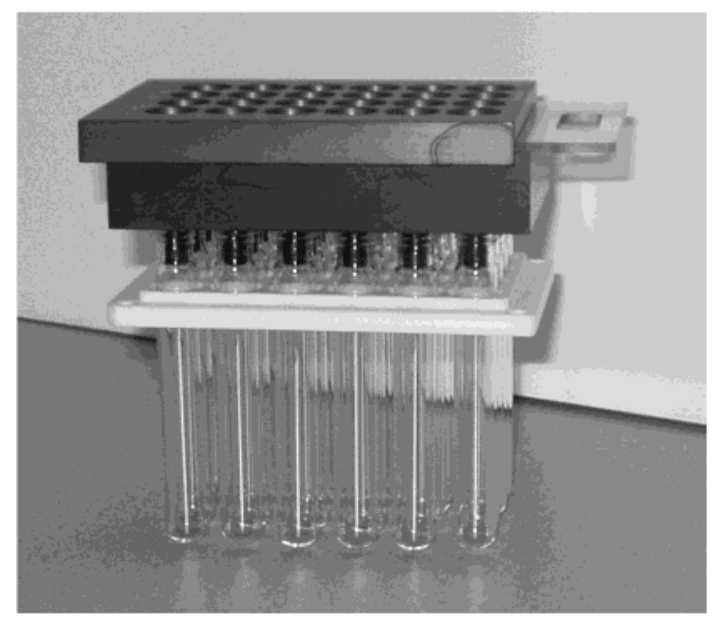

b. Solid Addition Plate with Funnel

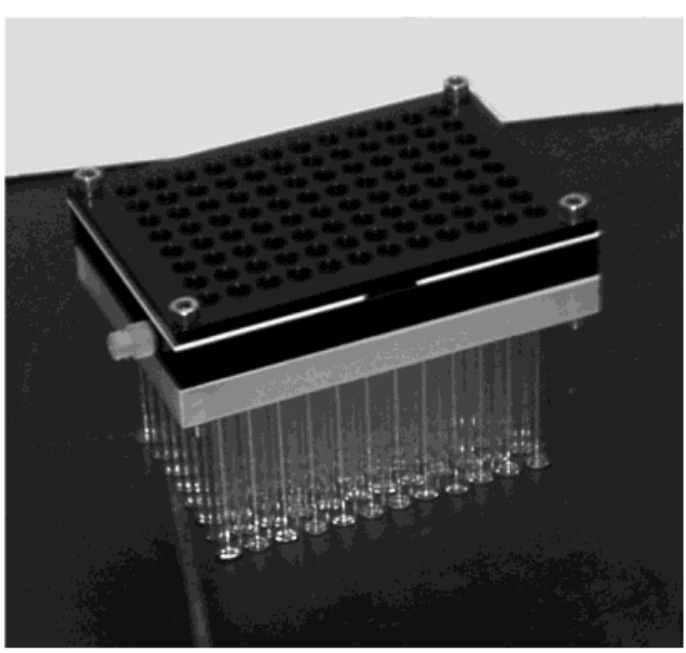

SynthArray-96

wear. The standard microtiter format also allows the facile integration of these reactors with a plethora of commercially available devices, including filter plates and liquid handling robots. Coupled with in-house automation of workups and purification technologies, the SynthArray-24 has enabled the facile production of numerous libraries for both lead generation and lead optimization projects. In summary, we believe the SynthArray design approaches the simplicity and functionality of the standard round-bottom flask toolkit but enables parallel synthesis in labs more accustomed to oneat-a-time chemistry.

Acknowledgment. We thank Kent Carrow and Warren Daniels of S \& D Machine and Tool, Inc. of Durham, NC, for consultation and manufacturing of the SynthArray 
reactors. We also thank the members of the Lead Generation Chemistry group of Lilly RTP for testing, use, and suggestions related to the development of the SynthArray reactors.

Supporting Information Available. Drawings of the SynthArray-24, including measurements, are available as Supporting Information. This material is available free of charge via the Internet at http://pubs.acs.org.

\section{References and Notes}

(1) (a) Devlin, J. P. High Throughput Screening: The Discovery of Bioactive Substances; Marcel Dekker: New York, 1997. (b) Jandeleit, B.; Schaefer, D. J.; Powers, T. S.; Turner, H. W.; Weinberg, W. H. Angew. Chem., Int. Ed. Engl. 1999, 38, 2494-2532.

(2) (a) DeWitt, S. H.; Schroeder, M. C.; Stankovic, C. J.; Strode, J. E.; Czarnik, A. W. Drug Dev. Res. 1994, 33, 116-124. (b) Gordon, E. M.; Kerwin, J. F., Jr. Combinatorial Chemistry and Molecular Diversity in Drug Discovery; Wiley: New York, 1998. (c) Xie, W.; Firstine, S. M. J. Comb. Chem. 2004, 6, 24-26.

(3) Listings of most commercially available equipment for combinatorial chemistry can be found on the web at sites such as http://www.combichemlab.com/website/files/home.htm and http://www.combinatorial.com/instruments.html.
(4) Detailed descriptions of the microtiter footprint, as defined by the Society for Biological Screening, can be found at http://sbsonline.com/msdc/index.html.

(5) Equipment parts are sometimes shown as partially transparent to help visualize how they work.

(6) Scott, W. L.; Schonegg, R. A.; Cwi, C. L. Vessel Handling System Useful for Combinatorial Chemistry. U.S. Patent 5,785,927; 1998.

(7) Model: IKAMAG RCT basic, IKA WORKS, Inc., 2635 Northchase Pkwy, SE, Wilmington, NC, 28405. Web site: http://www.ika.net/.

(8) Stirring systems for 96-well plates are available from V\&P Scientific, Inc., 9823 Pacific Heights Blvd., Suite T, San Diego, CA 92121. Web site: http://www.vp-scientific.com/. Orbital shakers with heating are available from J-KEM Scientific, Inc. 6970 Olive Blvd., St. Louis, MO 63130. Web site: http://www.jkem.com/.

(9) Evolution of the reactor design is aimed primarily at reducing manufacturing costs and increasing ease of use and reusability. For example, the machined lip described for the SynthArray-96 will not bear repeated use as well as the O-rings. However, an O-ring design would require custom O-rings at a considerable cost. A very similar and functional previous design, which required pouring silicone around the tubes to make a seal, was never embraced by chemists in our labs.

(10) Part No. 14-957-76A, Fisher Scientific International, Inc., Liberty Lane, Hampton, NH 03842.

CC0499643 